Commun. Korean Math. Soc. 28 (2013), No. 2, pp. 285-295

http://dx.doi.org/10.4134/CKMS.2013.28.2.285

\title{
A NOTE ON RADON-NIKODYM THEOREM FOR OPERATOR VALUED MEASURES AND ITS APPLICATIONS
}

\author{
NASIRUdDin AHMed
}

\begin{abstract}
In this note we present sufficient conditions for the existence of Radon-Nikodym derivatives (RND) of operator valued measures with respect to scalar measures. The RND is characterized by the Bochner integral in the strong operator topology of a strongly measurable operator valued function with respect to a nonnegative finite measure. Using this result we also obtain a characterization of compact sets in the space of operator valued measures. An extension of this result is also given using the theory of Pettis integral. These results have interesting applications in the study of evolution equations on Banach spaces driven by operator valued measures as structural controls.
\end{abstract}

\section{Introduction}

One of the most important topics in the theory of vector measures is the question of existence of Radon-Nikodym derivatives (RND) of one vector measure with respect to another. In particular, the question of existence of RND of a vector measure with respect to a nonnegative scalar measure is historically the most important one. It is well known that this classifies Banach spaces which have Radon-Nikodym Property (RNP) and those which lack this property. This property has major implications in the study of weak compactness in function spaces like $L_{1}(\mu, X)$ where $(\Omega, \Sigma, \mu)$ is any finite measure space. The most important ones are the celebrated Dunford theorem [2, Theorem 1 , p. 101] characterizing (relatively) weakly compact sets in $L_{1}(\mu, X)$ and the Bartle-Dunford-Schwartz theorem [2, Theorem 5, p. 105] characterizing (relatively) weakly compact sets in the space $M_{c a b v}(\Sigma, X)$ of countably additive $X$-valued vector measures having bounded variation. In both these theorems one requires RNP for both $X$ and its dual $X^{*}$. It is known that the dual of $L_{1}(\mu, X)$ is given by $L_{\infty}\left(\mu, X^{*}\right)$ if and only if $X^{*}$ has RNP with respect to $\mu$. Our interest here is to prove the existence of Radon-Nikodym derivatives of

Received February 29, 2012; Revised November 13, 2012.

2010 Mathematics Subject Classification. 28B05, 46B22, 46G10, 46A50.

Key words and phrases. space of operator valued measures, strong operator topology, Radon-Nikodym theorem, RNP, Bochner and Pettis integrals, evolution equations on Banach spaces. 
operator valued measures with respect to nonnegative scalar measures so that integration of vector valued functions with respect to operator valued measures can be simplified to integration of vector valued functions with respect to scalar measures.

These problems arise naturally in the study of control systems governed by differential equations on Banach spaces and driven by vector measures or operator valued measures [1].

Rest of the paper is organized as follows. In Section 2, we present briefly some well-known results on Radon-Nkiodym theorems in the context of Banach spaces including Maynrad's result which is closely related but different from the problem considered here. In Section 3, we present our main results. In Section 4, we present an extension of our main result based on Pettis integration. Using this result we extend the theory of Dobrakov integration to include operator valued measures having only finite scalar semivariation as opposed to semivariation. These results have interesting applications in the study of structurally controlled evolution equations on Banach spaces.

\section{Background and preliminaries}

Let $(D, \Sigma)$ be any measurable space with $\Sigma$ denoting the sigma algebra of subsets of the set $D$. Let $Z$ be any real Banach space and $M_{b a}(\Sigma, Z)$ the space of finitely additive $Z$ valued vector measures having bounded total variation. For any set function $\mu: \Sigma \longrightarrow Z$, we let $|\mu|(K)$ denote the variation of $\mu$ over the set $K \in \Sigma$ and $|\mu|(\cdot)$ the positive measure induced by the variation. $M_{b a}(\Sigma, Z)$ is a Banach space with respect to the norm topology induced by the variation $|\mu|_{v} \equiv|\mu|(D)$. The semivariation of $\mu$ over $E \in \Sigma$ is given by

$$
\|\mu\|_{s v}(E) \equiv \sup \left\{\left|z^{*} \mu\right|(E), z^{*} \in B_{1}\left(Z^{*}\right)\right\},
$$

where $Z^{*}$ denotes the (topological) dual of $Z, B_{1}\left(Z^{*}\right)$ the closed unit ball in $Z^{*}$ centered at the origin, and $\left|z^{*} \mu\right|(E)$ denotes the variation of the scalar measure over $E \in \Sigma$. The semivariation of $\mu$ over $D$ is then given by $\|\mu\|_{s v}(D)=$ $\sup \left\{\|\mu\|_{s v}(E), E \subset D, E \in \Sigma\right\}$. Clearly, it follows from the definition that $0 \leq\|\mu\|_{s v} \leq|\mu|_{v}$. The class of countably additive $Z$ valued vector measures denoted by $M_{c a}(\Sigma, Z)$ and equipped with the total variation norm is a closed linear subspace of $M_{b a}(\Sigma, Z)$ and hence it is also a Banach space. Similarly, for any pair of Banach spaces $\{X, Y\}, M_{b a}(\Sigma, \mathcal{L}(X, Y))$ will denote the space of finitely additive set functions defined on $\Sigma$ with values $\mathcal{L}(X, Y)$. For more on vector measures see $[2,5]$. For any real Banach space $X$, let $B_{\infty}(D, X)$ denote the Banach space of $X$ valued, norm bounded, $\Sigma$-measurable functions defined on $D$ endowed with the supnorm topology, $\|f\|_{\infty} \equiv \sup \left\{|f(s)|_{X}, s \in\right.$ $D\}$ for $f \in B_{\infty}(D, X)$, which are uniform limits of $\Sigma$-measurable $X$ valued simple functions. Let $\nu \in M_{c a b v}^{+}(\Sigma)$, the class of nonnegative countably additive measures having bounded variation, and $X$ any real Banach space and $\mu: \Sigma \longrightarrow$ $X$ a countably additive $\nu$-continuous bounded vector measure with bounded (total) variation. The measure $\mu$ is said to have Radon-Nikodym derivative 
with respect to the measure $\nu$ if there exists a Bochner integrable function $f: D \longrightarrow X$ such that for every $\sigma \subset D, \sigma \in \Sigma$,

$$
\mu(\sigma)=\int_{\sigma} f(s) \nu(d s) .
$$

A Banach space $X$ is said to have the Radon-Nikodym property (RNP) if, for every finite measure space $(D, \Sigma, \lambda)$ and every $\lambda$-continuous $X$ valued vector measure $m$ of bounded variation, there exists a $g \in L_{1}(\lambda, X)$ such that for every $\sigma \in \Sigma$

$$
m(\sigma)=\int_{\sigma} g(s) \lambda(d s) .
$$

In general one can consider the questions of existence of Radon-Nikodym derivatives of (i) a vector measure with respect to a (nonnegative) finite scalar measure, (ii) a vector measure with respect to another vector measure, (iii) a vector measure with respect to an operator valued measure.

It is interesting to note that the first one is most extensively studied in the literature leading to the origin of the notion of Banach spaces having RNP. The first two are special cases of the third (iii). It is the third one that was developed by Maynard in his celebrated paper [7]. Here he gives necessary and sufficient conditions for the existence of RND $f$ of a vector measure $m$ with respect to an operator valued measure $B ; d m=d B f$. In fact, Maynard's result covers all the three cases [Corollary (Rieffel [10]), p. 458], [Theorem 3.2, p. 459], [Theorem 3.3, p. 460], and [Theorem 3.4, p. 461].

The class of $X$ valued measurable functions $\{f\}$ introduced by Maynard consists of almost everywhere limits of Cauchy sequences of measurable simple functions. This is endowed with a norm topology given by

$$
\rho(f) \equiv\|f\|_{L_{X}^{o}(D, B)} \equiv\left|B_{f}\right|(D)
$$

which is just the total variation of the vector measure $B_{f}(\sigma) \equiv \int_{\sigma} B(d s) f(s)$, $\sigma \in \Sigma$, on $D$. This is a normed vector space (not a Banach space) denoted by $L_{X}^{o}(D, B)$. This class is much larger than the classical class $L_{X}^{1}(D, B)$ used by Dinculeanu where

$$
\|f\|_{L_{X}^{1}(D, B)} \equiv \int_{D}|f(s)|_{X}|B|(d s)
$$

which, however, is a Banach space. In this respect Maynard's result is much more general. For convenience of the reader we present the statement of Maynard's theorem below.

Theorem 2.1 ([7, Maynard, Theorem 2.1, p. 451]). Let $(D, \Sigma)$ be a measurable space with $D \in \Sigma$ and let $B: \Sigma \longrightarrow \mathcal{L}(X, Y)$ be an operator valued measure with finite total variation where $X$ and $Y$ are Banach spaces. Let $m: \Sigma \longrightarrow Y$ be a given measure. Then $m$ is the indefinite integral with respect to the operator valued measure $B$ of an $f \in L_{X}^{o}(D, B)$ if and only if

(m1): $m<<B$, 
$(\mathrm{m} 2):|m|(D)<\infty$,

(m3): for each $E \in \Sigma^{+} \equiv\{\sigma \in \Sigma:|B|(\sigma)>0\}$, there exists $F \subset E$, $F \in \Sigma^{+}$, and a compact set $K \subset X$ such that $F$ is localized in $K$ (See [7] for details).

Remark 2.2. In the context of Banach spaces, this is the most general result known so far. The author shows that Rieffel's result [10] on the Radon-Nikodym theorem for Bochner integral follows from this result as a special case. For this, one takes $B=\mu$ a scalar valued nonnegative measure having bounded total variation and $m$ a $Y$ valued vector measure having bounded variation. In this case $(\mathrm{m} 3)$ reduces to $(m 3)^{\prime}:$ for every $F \in \Sigma^{+}$there exists $E \subset F, E \in \Sigma^{+}$, such that the average range of $m$ on $E$ given by,

$$
A_{E}(m) \equiv\left\{m(\sigma) / \mu(\sigma), \sigma \subset E, \sigma \in \Sigma^{+}\right\},
$$

is a relatively compact subset of $Y$.

Further on, Maynard's result has been generalized by several authors to locally convex topological spaces. See Fernandez and Guerra [6] including the references therein.

Throughout the rest of the paper we let $L(X, Y)$ denote the space of linear operators (not necessarily bounded) from the Banach space $X$ to the Banach space $Y$, and $\mathcal{L}(X, Y)$ the space of bounded linear operators from $X$ to $Y$, a subset of $L(X, Y)$.

In this note, we consider the question of existence of Radon-Nikodym derivative of

$(\mathrm{P} 1)$ : an operator valued measure with respect to a nonnegative scalar measure.

The main reason for considering this problem is it's usefulness in the study of evolution equations on Banach spaces [1]. Unfortunately Maynard's result does not apply in this case even though our problem is much simpler than the problem considered by Maynard (Theorem 2.1).

There are other interesting problems like (P2): RND of an operator valued measure with respect to a vector measure, (P3): RND of an operator valued measure with respect to another operator valued measure. We do not consider these problems since we are not aware of any of their applications.

\section{A Radon-Nikodym theorem}

Here we consider problem $(\mathrm{P} 1)$. Let $M_{\text {casbv }}(\Sigma, \mathcal{L}(X, Y))$ denote the class of $\mathcal{L}(X, Y)$ valued vector measures which are countably additive in the strong operator topology having bounded variation. This is a linear subspace of the space of finitely additive operator valued measures $M_{b a}(\Sigma, \mathcal{L}(X, Y))$. We use the symbol $M_{c a b v}^{+}(\Sigma)$ to denote the class of countably additive nonnegative measures having bounded variation.

Theorem 3.1. Let $B \in M_{b a}(\Sigma, \mathcal{L}(X, Y))$ and $\mu \in M_{\text {cabv }}^{+}(\Sigma)$. Suppose (a1): $B$ is countably additive in the strong operator topology having bounded total 
variation, (a2): $|B|(\cdot)<<\mu(\cdot)$, and (a3): $Y$ has the Radon-Nikodym property. Then there exists a unique strongly measurable operator valued function $T$ : $D \longrightarrow \mathcal{L}(X, Y)$ which is strongly Bochner integrable such that

$$
B(\sigma) x=\int_{\sigma} T(s) x \mu(d s)
$$

for each $\sigma \in \Sigma$ and $x \in X$.

Proof. Let $x \in X$ and define the measure $m_{x}(\cdot) \equiv B(\cdot) x$. Since $B$ is countably additive in the strong operator topology having bounded variation, it is evident that $m_{x}$ is a countably additive $Y$ valued vector measure with bounded total variation. Clearly, it follows from assumption (a2) that $m_{x}$ is $\mu$ continuous. By assumption (a3), $Y$ has RNP (Radon-Nikodym Property) and hence there exists an $f_{x} \in L_{1}(\mu, Y)$ (Bochner integrable function) such that

$$
m_{x}(E) \equiv \int_{E} f_{x}(s) \mu(d s)
$$

for each $E \in \Sigma$. It is clear that $m_{\alpha x}=\alpha m_{x}$ and $m_{x_{1}+x_{2}}=m_{x_{1}}+m_{x_{2}}$ for every $\alpha \in R$ and $x_{1}, x_{2} \in X$. From this it follows that the map $x \in X \rightarrow f_{x}(s) \in Y$ is linear for all $s \in D$. Hence there exists a linear operator valued function $T$ : $D \longrightarrow L(X, Y)$ such that for every $x \in X, f_{x}(s)=T(s) x$ for all $s \in D$. Recall that $L(X, Y)$ denotes the class of linear operators from $X$ to $Y$ not necessarily bounded. Since $f_{x}$ is Bochner integrable, it is a strongly measurable $Y$ valued function and hence $T(\cdot)$ is strongly measurable $L(X, Y)$ valued function. That is, for each $x \in X, s \longrightarrow T(s) x$ is a strongly measurable $Y$ valued function and Bochner integrable on $D$ with respect to the measure $\mu$. Consequently, by definition, $T(\cdot)$ is $\mu$ integrable in the strong operator topology. Thus for each $x \in X,|T(s) x|_{Y}<\infty \mu$ a.e. Since by assumption (a1), $B$ has bounded total variation, $m_{x}(\cdot)$ has bounded total variation uniformly with respect to $x \in$ $B_{1}(X)$. Thus by virtue of Nikodym uniform boundedness principle [2, Theorem 1.3.1, p. 14], it follows from this that $\sup \left\{\left\|m_{x}\right\|(S), x \in B_{1}(X)\right\}<\infty$ for every $S(\subset D) \in \Sigma$. Then it follows from a well-known result [2, Proposition 1.1.11, p. 4] that $\sup \left\{\left|m_{x}(\sigma)\right|_{Y}, x \in B_{1}(X), \sigma \in \Sigma\right\}<\infty$. Hence, it follows from $\mu$ continuity of $m_{x}$ that, for $\mu$ almost all $s \in D, T(s) \in \mathcal{L}(X, Y) \subset L(X, Y)$. We prove uniqueness by contradiction. Suppose there are two such operator valued functions, $T_{1}, T_{2}$, giving the same operator valued measure $B$. In other words, for every $x \in X$ and $\sigma \in \Sigma$,

$$
m_{x}^{1}(\sigma) \equiv \int_{\sigma} T_{1}(s) x \mu(d s)=B(\sigma) x=\int_{\sigma} T_{2}(s) x \mu(d s) \equiv m_{x}^{2}(\sigma) .
$$

By hypothesis $B$ has bounded variation and therefore the measures $m_{x}^{1}$ and $m_{x}^{2}$ both have bounded variations and hence their difference $m_{x}^{1}-m_{x}^{2}$ has bounded variation. Since the variation of vector measures determined by the indefinite 
Bochner integrals, is given by the integral of the norm, it is clear that

$$
0=\left|m_{x}^{1}-m_{x}^{2}\right|(\sigma)=\int_{\sigma}\left|T_{1}(s) x-T_{2}(s) x\right|_{Y} \mu(d s)
$$

for every $\sigma \in \Sigma$ and $x \in X$. From this it is easy to argue that $T_{1}(s)=T_{2}(s)$ $\mu$-almost every where. This completes the proof.

Remark 3.2. Note that under the assumption (a1), we are in fact dealing with the smaller class $M_{\text {casbv }}(\Sigma, \mathcal{L}(X, Y))$ contained in $M_{\text {casbsv }}(\Sigma, \mathcal{L}(X, Y)$, (the space of operator valued measures countably additive in the strong operator topology having bounded semivariation) which, in turn, is contained in $M_{b a}(\Sigma, \mathcal{L}(X, Y))$.

Remark 3.3. It follows from Remark 2.2, that in Theorem 3.1, the assumption on $Y$ having RNP, (a3), can be relaxed by using the assumption (a3) $:$ for each $x \in X$ and each $E \in \Sigma^{+} \equiv\{\sigma \in \Sigma: \mu(\sigma)>0\}$ there exists $F \subset E, F \in \Sigma^{+}$, such that the average range of $m_{x}$ on $F$ given by

$$
A_{F}\left(m_{x}\right) \equiv\left\{m_{x}(\sigma) / \mu(\sigma), \sigma \subset F, \sigma \in \Sigma^{+}\right\}
$$

is a relatively compact subset of $Y$. Thus it follows from Rieffel's theorem [10, Main Theorem, p. 466] that the assumptions (a1), (a2) and $\overline{(\mathrm{a} 3)}$ are both necessary and sufficient.

Next we consider certain topological properties of operator valued measures satisfying the assumptions of Theorem 3.1 or those of Remark 3.3.

Let $X, Y$ be a pair of real Banach spaces, and $\mathcal{L}(X, Y)$ the space of bounded linear operators from $X$ to $Y$. Let $\mathcal{M}_{\mu}(\Sigma, \mathcal{L}(X, Y))\left(\subset M_{\text {casbv }}(\Sigma, \mathcal{L}(X, Y))\right)$ denote the class of operator valued measures satisfying the conditions (a1)(a3) of Theorem 3.1 (or (a1), (a2), $\overline{(\mathrm{a} 3)}$ ). We introduce the vector space $L_{1}\left(D_{\mu}, \mathcal{L}(X, Y)\right)$ consisting of strongly measurable (in the strong operator topology) operator valued functions defined on $D$ which are (strongly) Bochner integrable with respect to the measure $\mu$ satisfying

$$
\sup \left\{\left|\int_{\sigma} T(s) x \mu(d s)\right|_{Y}, x \in B_{1}(X)\right\}<\infty
$$

for every $\sigma \in \Sigma$. We can furnish this vector space with a seminorm as follows. For each $E \in \Sigma$, let $\Pi_{\Sigma}(E)$ denote the class of all finite disjoint $\Sigma$-measurable partitions of the set $E$. Define

$$
|T|_{\mu}(E) \equiv \sup _{\pi \in \Pi_{\Sigma}(E)} \sum_{\sigma \in \pi} \sup \left\{\left|\int_{\sigma} T(s) x \mu(d s)\right|_{Y}, x \in B_{1}(X)\right\}
$$

and $\|T\|_{\mu}=\sup \left\{|T|_{\mu}(E), E \in \Sigma\right\}$. The reader can easily verify that this defines an equivalence class in the sense that $T_{1}=T_{2}$ if and only if $T_{1}(s) x=T_{2}(s) x$ $\mu$ a.a $s \in D$ and $x \in X$. Identifying elements in the equivalence class, it is easy to see that this defines a norm $\left(\|T\|_{\mu}\right)$ on $L_{1}\left(D_{\mu}, \mathcal{L}(X, Y)\right)$. As a corollary of Theorem 3.1, we have the following result. 
Corollary 3.4. Under the assumptions of Theorem 3.1, the correspondence $B \leftrightarrow T$, defines an isometric isomorphism between the spaces $\mathcal{M}_{\mu}(\Sigma, \mathcal{L}(X, Y))$ and $L_{1}\left(D_{\mu}, \mathcal{L}(X, Y)\right)$, with the later space endowed with the norm topology as described above.

In fact we have more. Suppose $M_{\text {casbv }}(\Sigma, \mathcal{L}(X, Y))$ is endowed with the topology of set wise convergence in the strong operator topology of $\mathcal{L}(X, Y)$. This is a locally convex sequentially complete topological vector space. In the following proposition we prove that $\mathcal{M}_{\mu}(\Sigma, \mathcal{L}(X, Y))$ is a closed linear subspace of $M_{\text {casbv }}(\Sigma, \mathcal{L}(X, Y))$.

Proposition 3.5. The set $\mathcal{M}_{\mu}(\Sigma, \mathcal{L}(X, Y))$ is a closed linear subspace of $M_{\text {casbv }}(\Sigma, \mathcal{L}(X, Y))$ with respect to the topology of setwise convergence in the strong operator topology.

Proof. It is clear that $\mathcal{M}_{\mu}(\Sigma, \mathcal{L}(X, Y))$ is a linear subspace of $M_{\text {casbv }}(\Sigma, \mathcal{L}(X$, $Y))$. We prove that it is closed. Consider the sequence $\left\{B_{n}\right\} \in \mathcal{M}_{\mu}(\Sigma, \mathcal{L}(X, Y))$ and suppose that $B_{n}$ converges to $B_{o}$ set wise on $\Sigma$ in the strong operator topology. Our concern is to verify that $B_{o} \in \mathcal{M}_{\mu}(\Sigma, \mathcal{L}(X, Y))$. By definition of set wise convergence in the strong operator topology, we have, for each $\sigma \in \Sigma$ and $x \in X$,

$$
m_{n}^{x}(\sigma) \equiv B_{n}(\sigma) x \stackrel{s}{\longrightarrow} B_{o}(\sigma) x \equiv m_{o}^{x}(\sigma) \text { in } Y .
$$

Since for each $n \in N, B_{n}$ is countably additive in the strong operator topology and $\mu$ continuous, $m_{n}^{x}$ is countably additive and $\mu$ continuous, and set wise convergent to the $Y$-valued vector measure $m_{o}^{x}$. Thus by the well-known Vitali-Hahn-Saks-Nikodym theorem (see $[2,5]) m_{o}^{x}$ is countably additive and that countable additivity is uniform and $m_{o}^{x}$ is $\mu$ continuous. Thus $B_{o}$ is countably additive in the strong operator topology and $\mu$-continuous. Since $M_{\text {casbv }}(\Sigma, \mathcal{L}(X, Y))$ is sequentially complete, the limit $B_{o} \in M_{\text {casbv }}(\Sigma, \mathcal{L}(X, Y))$ and consequently $B_{o}$ has also bounded variation. Thus $B_{o}$ satisfies (a1)-(a2) of Theorem 3.1 and $Y$ has RNP, and hence $B_{o} \in \mathcal{M}_{\mu}(\Sigma, \mathcal{L}(X, Y))$. This completes the proof.

In the study of optimization and optimal control, compactness plays a crucial role. We present here a simple result characterizing compact sets in $\mathcal{M}_{\mu}(\Sigma, \mathcal{L}(X, Y))$

Theorem 3.6. Let $\Gamma$ be a subset of $\mathcal{M}_{\mu}(\Sigma, \mathcal{L}(X, Y))$ and suppose it satisfies the following conditions: (C1): $\Gamma$ is bounded, (C2): for each $x \in X$ and $\sigma \in \Sigma$, the set $\{B(\sigma) x, B \in \Gamma\}$ is a relatively compact subset of $Y$. Then $\Gamma$ is relatively sequentially compact with respect to set wise convergence in the strong operator topology.

Proof. Let $\left\{B_{n}\right\} \in \Gamma$. For each $x \in X$, define the sequence of $Y$ valued vector measures $\left\{m_{x}^{n}\right\}$ given by $m_{x}^{n}(\sigma) \equiv B_{n}(\sigma) x$ for $\sigma \in \Sigma$. Since $\Gamma \subset$ $\mathcal{M}_{\mu}(\Sigma, \mathcal{L}(X, Y))$, it follows from assumption (C1) that this is a sequence of bounded countably additive $Y$-valued measures. By assumption (C2), for each 
$\sigma \in \Sigma$ and $x \in X$, there exists a subsequence of the sequence $\left\{m_{x}^{n}(\sigma)=\right.$ $\left.B_{n}(\sigma) x\right\}$ such that $m_{x}^{n_{k}}(\sigma)=B_{n_{k}}(\sigma) x \stackrel{s}{\longrightarrow} y_{x}^{o}$ for some $y_{x}^{o} \in Y$. Then by virtue of Banach-Steinhaus theorem there exists a $B_{o}(\sigma) \in \mathcal{L}(X, Y)$ such that $y_{x}^{o}=B_{o}(\sigma) x$. This way we can construct an operator valued set function defined on each $\sigma \in \Pi$, where $\Pi$ is any finite disjoint $\Sigma$-measurable partition of $D$. Let $\Pi_{\Sigma}(D)$ denote the class of all finite disjoint $\Sigma$-measurable partitions of $D$. We can introduce a partial order on this class turning it into a partially ordered set $\left(\Pi_{\Sigma}(D), \leq\right)$ as follows. For every $k \in N$, let $\Pi_{k} \in \Pi_{\Sigma}(D)$ and call $\Pi_{k} \leq \Pi_{k+1}$ if every set in $\Pi_{k}$ is given by a finite union of sets from $\Pi_{k+1}$. Then by use of this sequence or chain $\left\{\Pi_{k}, k \in N\right\}$ and Zorn's lemma, one can construct a set function $\tilde{B}_{o}: \Sigma \longrightarrow \mathcal{L}(X, Y)$ giving an operator valued measure. Define for each $x \in X$ the vector measure $m_{x}^{o}$ by setting $m_{x}^{o}(\sigma)=\tilde{B}_{o}(\sigma) x$. Note that $m_{x}^{o}(\sigma)$ is the set wise strong (norm) limit of the sequence $\left\{m_{x}^{n_{k}}(\sigma)\right\}$ and so by Vitali-Hahn-Saks-Nikodym theorem $m_{x}^{o}$ is countably additive and $\mu$ continuous. So $\tilde{B}_{0}$ is countably additive in the strong operator topology and $\mu$-continuous and by virtue of Nikodym Uniform boundedness principle it is bounded. Thus $\tilde{B}_{o} \in \mathcal{M}_{\mu}(\Sigma, \mathcal{L}(X, Y))$ proving relative sequential compactness of $\Gamma$.

Remark 3.7. It follows from Corollary 3.4 and Theorem 3.6 that under the isomorphism $B \leftrightarrow T$ denoted by $\Phi$, a set $V \subset L_{1}\left(D_{\mu}, \mathcal{L}(X, Y)\right)$ is relatively sequentially compact if and only if $\Phi^{-1}(V)$ is relatively sequentially compact in $\mathcal{M}_{\mu}(\Sigma, \mathcal{L}(X, Y))$ and conversely.

\section{An extension of Theorem 3.1}

Integration of vector valued functions with respect to operator valued measures was extensively studied by Dobrakov in a series of papers [3, 4]. For an excellent review on the subject see Panchapagesan [9] (also see the references therein). Dobrakov considers a larger class of operator valued measures denoted by $M_{\text {casbsv }}(\Sigma, \mathcal{L}(X, Y))$ as mentioned in Remark 3.2. These operators have only bounded semivariations which are majorized by their variations. This is a weaker condition. We can extend our Theorem 3.1 to include this case.

Theorem 4.1. Let $B \in M_{b a}(\Sigma, \mathcal{L}(X, Y))$ and $\mu \in M_{\text {cabv }}^{+}(\Sigma)$. Suppose

(e1) for every $x \in X, m_{x}(\cdot) \equiv B(\cdot) x<<\mu(\cdot)$,

(e2) for every $\varepsilon>0$ there exists $D_{\varepsilon} \subset D, D_{\varepsilon} \in \Sigma$ with $\mu\left(D \backslash D_{\varepsilon}\right)<\varepsilon$ such that, for every $x \in X$, the set

$$
Y_{\varepsilon} \equiv\left\{m_{x}(\sigma) / \mu(\sigma): \sigma \subset D_{\varepsilon}, \sigma \in \Sigma, \mu(\sigma)>0\right\}
$$

is a relatively weakly compact subset of $Y$. Then there exists a unique strongly measurable operator valued function $T: D \longrightarrow \mathcal{L}(X, Y)$ which is Pettis integrable in the strong operator topology, so that, for every $x \in X$,

$$
m_{x}(E)=\mathcal{P}-\int_{E} T(s) x \mu(d s) \in Y
$$


for every $E \in \Sigma$. In other words, for each $y^{*} \in Y^{*}, \int_{E}\left|y^{*}(T(s) x)\right| \mu(d s)<\infty$ for each $E \in \Sigma$ and $x \in X$.

Proof. We present a brief outline of the proof. Under the assumptions (e1) and (e2), it follows from Theorem 2 of Moedomo and Uhl, Jr. [8, Theorem 2 , p. 534] that, for every $x \in X$, there exists a strongly measurable Pettis integrable function $f_{x}$ such that

$$
m_{x}(E)=\mathcal{P}-\int_{E} f_{x}(s) \mu(d s) \in Y
$$

for every $E \in \Sigma$. Then it follows from similar arguments as in the proof of Theorem 3.1, that $x \rightarrow f_{x}(s)$ is a linear map from $X$ to $Y$ for $\mu$ almost all $s \in D$. Hence there exists an operator valued function $T: D \rightarrow \mathcal{L}(X, Y)$ measurable in the strong operator topology such that

$$
m_{x}(E)=\mathcal{P}-\int_{E} f_{x}(s) \mu(d s)=\mathcal{P}-\int_{E} T(s) x \mu(d s)
$$

for every $x \in X$. To prove uniqueness, we assume to the contrary and suppose there are two such distinct operator valued functions $T_{1}, T_{2}$ generating the same vector measure $m_{x}$ for each $x \in X$. Then, it follows from the above expression that, for every $E \in \Sigma$ and $y^{*} \in Y^{*}$, we have

$$
y^{*}\left(\mathcal{P}-\int_{E}\left[T_{1}(s) x-T_{2}(s) x\right] \mu(d s)\right)=\int_{E} y^{*}\left(T_{1}(s) x-T_{2}(s) x\right) \mu(d s)=0
$$

for each $x \in X$. Using density argument and Hahn-Banach theorem, it is easy to verify from the above expression that $T_{1}(s) x=T_{2}(s) x \mu$-almost all $s \in D$ and for every $x \in X$ implying that $T_{1}(s)=T_{2}(s) \mu$-almost every where in the strong operator topology. This contradiction completes the proof.

There are two interesting implications of the above theorem:

(I) It is well known that the measure induced by the indefinite Pettis integral is countably additive and so bounded. Thus under the assumptions of the above theorem, the operator valued measure $B$ must be necessarily countably additive in the strong operator topology. Further, by the uniform boundedness theorem, it is bounded.

(II) By use of closed graph theorem, one can verify that the map $L: Y^{*} \longrightarrow$ $L_{1}(\mu)$, given by $y^{*} \longrightarrow y^{*}(T(\cdot) x)$ ), is continuous and hence bounded. Using this fact, it is not difficult to verify that, for each $x \in X$, the measure $m_{x}$ has finite semivariation, (that is $\left\|m_{x}\right\|(D)<\infty$ ) (but not necessarily finite variation). On the other hand, it follows from straightforward computation using the definition of scalar semivariation [9] of operator valued measures that, for every $\sigma \in \Sigma$,

$$
\sup \left\{\left\|m_{x}\right\|(\sigma), x \in B_{1}(X)\right\} \leq|B|_{s s v}(\sigma) .
$$

Further, it is well known [9] (see also [3, 4]) that, for every $\sigma \in \Sigma,|B|_{s s v}(\sigma) \leq$ $|B|_{s v}(\sigma) \leq|B|_{v}(\sigma)$, where $|B|_{s s v}(\sigma),|B|_{s v}(\sigma)$ and $|B|_{v}(\sigma)$ denote respectively 
the scalar semivariation, the semivariation, and the variation of the operator valued measure $B$ on $\sigma$ implying that the embeddings $M_{\text {casbv }}(\Sigma, \mathcal{L}(X, Y)) \hookrightarrow$ $M_{\text {casbsv }}(\Sigma, \mathcal{L}(X, Y)) \hookrightarrow M_{\text {casbssv }}(\Sigma, \mathcal{L}(X, Y))$ are continuous.

Recall that the theory of Dobrakov integral [3] of a vector valued function with respect to an operator valued measure is based on the assumption that the operator valued measure is countably additive in the strong operator topology and has bounded semivariation. This is the class in the middle. Apparently, the inequality (1) shows that Theorem 4.1 allows a larger class of operator valued measures than those used for the Dobrakov theory of integration $[3,4,9]$. It suffices if the operator valued measure has only finite scalar semivariation, a weaker condition than finite semivariation. More precisely we have the following result.

Corollary 4.2. For the space of operator valued measures $M_{\text {casbssv }}(\Sigma, \mathcal{L}(X$, $Y)$ ), there exists a nontrivial closed linear subspace $\mathcal{G} \subset B_{\infty}(D, X)$ with norm $|f|_{\mathcal{G}}$ such that, for every $B \in M_{\text {casbssv }}(\Sigma, \mathcal{L}(X, Y))$ and every $f \in \mathcal{G}$, the integral

$$
G_{B}(f) \equiv \int_{D} B(d s) f(s) \in Y
$$

is a well defined element of $Y$ and $\left|G_{B}(f)\right|_{Y} \leq|B|_{\text {ssv }}|f|_{\mathcal{G}}$.

Acknowledgement. (i) The author thanks the reviewers for their valuable suggestions. (ii) Thanks to the National Science and Engineering Research Council of Canada for supporting the research under grant no A7109.

\section{References}

[1] N. U. Ahmed, Vector and Operator Valued Measures as Controls for Infinite Dimensional Systems: Optimal Control, Discuss. Math. Differ. Incl. Control Optim. 28 (2008), 95-131.

[2] J. Diestel and J. J. Uhl, Jr., Vector Measures, American Mathematical Society, Providence, R.I., 1977.

[3] I. Dobrakov, On integration in Banach spaces I, Czechoslovak Math. J. 20(95) (1970), 511-536.

[4] - On Integration in Banach Spaces IV, Czechoslovak Math. J. 30(105) (1980), no. 2, 25979 .

[5] N. Dunford and J. T. Schwartz, Linear Operators, Part 1, General Theory, Second Printing, Interscience Publishers, Inc., New York, 1964.

[6] F. J. Fernandez and P. J. Guerra, On the Radon-Nikodým property for operator-valued measures, Period. Math. Hungar. 22 (1991), no. 3, 147-151.

[7] H. B. Maynard, A Radon-Nikodým theorem for operator-valued measures, Trans. Amer. Math. Soc. 173 (1972), 449-463.

[8] S. Moedomo and J. J. Uhl, Jr., Radon-Nikodym theorems for the Bochner and Pettis integrals, Pacific J. Math. 38 (1971), no. 2, 531-536.

[9] T. V. Panchapagesan, On the distinguishing features of the Dobrakov integral, Divulg. Mat. 3 (1995), no. 1, 79-114.

[10] M. A. Rieffel, The Radon-Nikodym theorem for the Bochner integral, Trans. Amer. Math. Soc. 131 (1968), 466-487. 
UNIVERSITY OF OTTAWA

OtTaWa K1N6N5, Canada

E-mail address: ahmed@site.uottawa.ca 